\title{
Aplicaciones de los blogs en unidades de información: usos y perspectivas
}

\author{
Isabel Andreu Felipe (1)
}

Carmen María Brugarolas Ros (2)

Concepción Dolores Alcázar Ruiz (1)

José Luis Cárceles Andreu (3)

(1) Servicio Regional de Empleo y Formación (Murcia, España)

(2) Biblioteca Universitaria de Murcia (España)

(3) Instituto de la Mujer (Murcia, España)

\section{Resumen}

El presente artículo analiza el concepto de weblog, su estructura y el uso que se hace de ellos en la actualidad. A partir de los usos conocidos y analizando las funcionalidades que ofrece cada uno de los componentes de esta herramienta, se compara con las necesidades comunicativas de las unidades de información y se exponen propuestas.

Palabras clave: Weblogs. Sindicación de contenidos. Bibliotecas. Unidades de información.

\section{Abstract}

The concept of weblog, its structure and its use in the context of libraries and information units are analyzed. First, the functionalities and uses of blogs are described and studied. Later, the potential utility of these functionalities for different libraries and information units is explored.

Keywords: Weblogs. RSS. Libraries. Information units.

\section{Marco teórico}

Los weblogs nacen y crecen en la Red, por lo que Internet es el mejor lugar para encontrar datos sobre estos, aunque, con la popularidad que han alcanzado, las fuentes de información se multiplican. Prueba de ello es que podemos encontrar artículos sobre la blogosfera (conjunto de weblogs y sus relaciones) incluso en revistas de divulgación científica como Muy Interesante, magazines como Mía o El Semanal y diarios de información general como El Mundo, El País, ABC, etcétera. Se puede afirmar que es un asunto extendido en el ámbito informativo. 
Entre los diversos autores que se han consultado para la investigación bibliográfica es necesario destacar algunos por sus importantes aportaciones al mundo de los weblogs.

Rebecca Blood fue pionera al publicar una monografía sobre weblogs en 2002: The weblog handbook: practical advice on creating and maintaining your blog. Es experta en weblogs a nivel mundial, tiene varios libros editados y mantiene un weblog, Rebecca's pocket, donde se pueden encontrar sus artículos sobre el tema.

En el ámbito hispanoparlante tenemos la referencia de José Luis Orihuela, profesor de la Facultad de Comunicación de la Universidad de Navarra, considerado una de las personas más influyentes de la blogosfera hispana. Ha publicado artículos en revistas de distintas disciplinas (comunicación, educación, periodismo...) y además utiliza los weblogs como herramienta para la docencia. Su weblog eCuaderno mantiene un puesto destacado en el conjunto de los blogs hispanos.

Por otra parte, Laurel A. Clyde, profesora de la Facultad de Ciencias Sociales de la Universidad de Islandia, investiga actualmente sobre el uso de Internet y los servicios de información en línea en distintos campos. Ha publicado varios libros y artículos en revistas nacionales e internacionales. Fue webmáster de la Asociación Internacional de Bibliotecarios Escolares durante diez años, y mantiene un sitio web sobre weblogs. En 2004 publicó la monografía Weblogs and libraries, donde se hace un buen compendio del estado de la cuestión, aunque orientado hacia las bibliotecas. Esta desviación de lo general a lo particular nos interesa, ya que dejamos atrás las aplicaciones más "domésticas" de los weblogs para centrarnos en su uso como fuentes y como divulgadores de la información.

\subsection{Concepto y denominaciones}

Weblog (o su nombre abreviado, blog), cuaderno de bitácora o simplemente bitácora son todas las denominaciones que se le han dado; el más usado en la actualidad es blog, y de él derivan una serie de palabras como blogger (escritor de blogs), blogging (acción de escribir en un blog), blogosfera (conjunto de blogs y sus relaciones), etcétera.

Una bitácora es una especie de diario en el que los capitanes de barco anotan las coordenadas de su ruta y los avatares de su travesía. Por similitud de ideas se empezó a utilizar este término. Los mexicanos Carlos Tirado y Gustavo Arizpe estuvieron entre los primeros autores de weblogs en español, y fueron los responsables de la popularización del término bitácora, hoy utilizado por millones de personas.

Rebecca Blood (2002) afirma que es como una conversación entre diferentes personas. No deja de ser una página web en la que hay entradas (mensajes) ordenadas por fecha, empezando por las más recientes. Los visitantes pueden opinar sobre los temas expuestos y escribir en la misma página su mensaje, que quedará registrado para que los demás puedan leerlo. 
Para Jane Perrone (2004), un weblog es, literalmente, un $\log$ (registro) de la web: un sitio estilo diario donde el autor (el blogger) enlaza con otras páginas web que encuentra interesantes a través de entradas o mensajes colocados en orden cronológico inverso.

El canadiense Peter Scott (2005), experto en weblogs de la Biblioteca de la Universidad de Saskatchewan (Canadá), ha desarrollado una definición muy abierta de blog o weblog: "A web page containing brief, chronologically arranged items of information".

Por su parte, Jaime Alonso y Lourdes Martínez (2003) los definen mediante estos rasgos: $a$ ) es un espacio de comunicación personal; $b$ ) sus contenidos abarcan cualquier tipología; c) los contenidos presentan una marcada estructura cronológica; $d$ ) el sujeto que las elabora suele adjuntar enlaces a sitios web que tienen relación con los contenidos que se desarrollan; $e$ ) la interactividad aporta un alto valor añadido como elemento dinamizador en el proceso de comunicación.

\subsection{Tipos y clasificación}

Los escritores de weblogs no son muy amigos de clasificar la blogosfera debido a que se encuentra en pleno crecimiento. Hay muchísimos weblogs que son inclasificables en su conjunto, sobre todos los personales; esta situación ya se ha puesto de manifiesto en los directorios de páginas web. Para salvar estas dificultades se dan distintas propuestas, como aplicar una clasificación temática a los mensajes (post) concretos y una formal a los weblogs. Un ejemplo sería un fotoblog sobre arte urbano, o una clasificación de los weblogs por temas (bases de datos, diseño, cocina, política...) y enfoque, que es la forma de tratar el tema (opinión, información factual, referencia a sitios web descubiertos, sondeo...). Debe tratarse de clasificaciones abiertas y flexibles.

Wikipedia.com, la enciclopedia hecha por los internautas, ofrece esta taxonomía: Personales

Pensamientos, reflexiones.

Blogs de varios amigos que forman una red de trabajo distribuida (llamados friendblogs)

Temáticos

Noticias

Política

Temas legales (blawgs)

Periodísticos

Literarios

Religiosos

Colaborativos (también llamados colectivos o grupales)

Documentos elaborados por múltiples usuarios como wikipedia.com

Scire. $13: 1$ (en.-jun. 2007) 133-144. ISSN 1135-3716. 
De uso educativo

Directorios

Corporativos

Anuncios, consejos, etcétera

Formatos

Audio

Fotografía

Vídeo

Laurel A. Clyde (2004, p. 10) propone agruparlos por contenido, formato, propósito, modo en el que han sido creados, modo de acceso (público o privado) y características del autor.

\subsection{Principales estructuras}

Para profundizar en el estudio de los weblogs conviene analizar su estructu$\mathrm{ra}$, que suele ser similar, en parte debido a que muchos comparten el mismo software. En la parte principal de la página se encuentra el último tema tratado y, debajo, los comentarios de los visitantes, ya sea en la misma pantalla o en otra. Es posible consultar y buscar los temas tratados con anterioridad, que generalmente están colocados en orden cronológico inverso. Naturalmente, incluyen enlaces a otras bitácoras e información sobre el autor, y suelen ofrecer un servicio de sindicación de contenidos.

En cuanto a su contenido y finalidad, pueden ser cualquiera que sirva al autor como modo de expresión: textos literarios, ensayos, textos científicos, critica de productos, política, fotografías, vídeos, sonidos, dibujos... Es decir, se mezclan formatos y temas. También los propósitos pueden ser de lo más variopinto cuando hablamos de diarios personales. Cuando, por el contrario, el weblog pertenece a una colectividad (llámese asociación, empresa o grupo de investigación) o sigue siendo unipersonal pero de tipo profesional, los temas tratados suelen ser de actualidad y en muchos casos vienen motivados por alguna noticia aparecida en la prensa general o en revistas científicas. Otras veces se trata de una inquietud en la comunidad científica/profesional de la cual el blogger ha tenido noticia, o simplemente se le ocurre compartir un pensamiento. Si se trata de un weblog corporativo, el propósito del mismo suele ser la difusión de información de la empresa, ya sea de carácter interno o externo.

\subsection{Sindicación de contenidos}

Otra característica de los weblogs es la posibilidad de tener en nuestro poder las últimas informaciones publicadas en nuestros sitios favoritos de manera rápida y fácil. Son tantos los que nos pueden interesar que resultará una empresa casi imposible estar visitando periódicamente su dirección web para comprobar las últimas actualizaciones. 
Really Simple Syndication (RSS) es un formato basado en XML que constituye un nuevo modo de distribuir contenidos de un sitio web a los usuarios que lo requieran sin que tengan que entrar ellos mismos en la página, algo así como un servicio de alerta de novedades, pero lo que recibe el usuario es el contenido en sí mismo. Sindicar es publicar una noticia en diversos sitios. En palabras de Cambronero (2004),

Es un avance muy significativo, pues la información puede extraerse de sus repositorios originales y situarse en cualquier otro lugar, bien sea en otra página web o, incluso, en un reproductor multimedia. Hoy, en un iPod (el reproductor portátil de música de Apple) se pueden grabar ficheros de audio mp3, provenientes de weblogs, utilizando RSS.

Para poder acceder a este servicio es necesario tener un lector de noticias (en terminología bloguera se les llama feeds) o agregador de canales de sindicación. Permite suscribirse a un canal de sindicación determinado (un sitio web), y así recibir toda la información nueva que se publique en este. Existen varias maneras de recibir feeds: por correo electrónico, por Internet o por medio de un programa instalado en nuestro PC. El proceso de agregación es complementario del de sindicación. Al incorporar un weblog a nuestro lector de noticias, cada vez que se ponga en marcha el programa rastreará la Red en busca de actualizaciones.

\section{Marco práctico}

\subsection{Funciones}

Rebeca Blood (2002) afirma que los weblogs proporcionan contexto a la información, promueven la cultura, ofrecen puntos de vista alternativos, invitan a la participación e incitan a evaluar la información.

Merlo y Sorli (2003) los consideran unos excelentes recursos informativos, complementarios de las listas de distribución, grupos de noticias, chats, directorios y buscadores, e incluso de las revistas profesionales.

En el ámbito científico/profesional pueden constituir una revolución, ya que el contenido de un weblog puede comentar un artículo; a ese comentario, los visitantes pueden añadir otros, y así va construyendo un corpus teórico, "desgranando" las bases de la ciencia. Es una manera de interpretarla que permite compartir opiniones y conocimiento.

En opinión de José Luis Orihuela (2005),

Las funciones de la blogosfera en el nuevo escenario comunicativo son múltiples: es un filtro social de opiniones y noticias, un sistema de alerta temprana para los medios, un sistema de control y crítica de los medios, un factor de movilización social, un nuevo canal para las fuentes reconvertidas en medios, un nuevo formato aplicable en las versiones electrónicas de los medios tradicionales para coberturas de continuidad,

Scire. $13: 1$ (en.-jun. 2007) 133-144. ISSN 1135-3716. 
catástrofes y accidentes, un gigantesco archivo que opera como memoria de la Web, el alimento privilegiado de los buscadores por su renovación constante y su alta densidad de enlaces de entrada y de salida y, finalmente, es la gran conversación de múltiples comunidades cuyo anclaje común es el conocimiento compartido.

\subsection{Usos y aplicaciones más frecuentes en unidades de información}

En cuanto a las aplicaciones de la sindicación de contenidos, hemos de decir que es un servicio que han popularizado las bitácoras y, si bien es ya una característica propia de ellos, no lo es en exclusiva.

Actualmente es raro el diario que en su edición digital no ofrece un servicio de sindicación de contenidos para sus noticias. Algunos repositorios de documentos, como E-LIS (E-Prints in Library and Information Science), también lo utilizan para mantener al día a la comunidad científica. Simplemente por el ahorro de tiempo que supone al investigador este servicio de alerta ya es una herramienta que se debe tener en cuenta. Es como un servicio de resúmenes con un coste prácticamente nulo. Desde este punto de vista sería interesante estudiar todas las posibles utilidades de la sindicación de contenidos. Mediante esta tecnología, las editoriales y/o distribuidoras de revistas científicas pueden informar a sus lectores de los sumarios de los últimos números publicados, y las bibliotecas, de los últimos números recibidos.

Así, las bibliotecas pueden dar noticia de las últimas adquisiciones sin apenas esfuerzo. Esto ya lo hacen algunas de ellas. Para llevar a cabo esta tarea con comodidad es conveniente que las aplicaciones de gestión bibliotecaria permitan la exportación de los datos del catálogo al formato adecuado para publicar en Internet, que será el XML. Ya existen herramientas para para la conversión de registros en otros formatos a XML.

Como vemos, a través de los weblogs podemos sistematizar y actualizar nuestro sitio web a la vez que ofrecemos los servicios tradicionales. Hemos agrupado por áreas de trabajo algunos de los usos más importantes en unidades de información:

- Actividades de difusión de la colección y la biblioteca: difusión de información general sobre los servicios de las unidades de información; servicio de alerta de nuevas adquisiciones y difusión selectiva de la información (DSI); acceso a toda nuestra biblioteca digital.

- Servicios de información bibliográfica y de referencia: información bibliográfica on-line, incluyendo la "referencia virtual"; suministro de enlaces temáticos de interés; acceso a colecciones de referencia (información rápida mediante diccionarios, enciclopedias, repertorios, anuarios, bases de datos, etcétera); formación de usuarios.

- Servicios de información especializada y/o local: información sobre noticias profesionales y académicas a modo de tablón de anuncios virtual; servicio de alerta de información local, como convocatorias, eventos, conciertos, et- 
cétera (información de rápida obsolescencia); información sobre jornadas, congresos o cursos de interés.

- Comunicación de/con los usuarios: un foro para que los usuarios puedan opinar y comunicarse o para, por ejemplo, debatir lecturas de los usuarios; un espacio para poder resolver dudas, a modo de "referencia virtual" donde el usuario pregunta y realiza sus consultas; recepción de desideratas.

Estas son tan solo algunas ideas de las aplicaciones y los usos que en el momento actual podemos poner en práctica para optimizar nuestras tareas y servicios, integrando así en nuestro quehacer diario las nuevas tecnologías que se nos ofrecen.

\subsection{Ejemplos prácticos}

Hemos hecho una revisión, sin ánimo de ser exhaustivos, tan solo con el propósito de mostrar un par de ejemplos de cómo los weblogs en España se van integrando cada día como una herramienta más en las páginas web de nuestros centros. Teniendo en cuenta, eso sí, que los weblogs en castellano referentes al campo de la biblioteconomía y la documentación no son numerosos aún, y casi todos tienen menos de un año de antigüedad.

\section{Biblioteca Pública de Pozo Cañada} http://bibliopozoca.blogspot.com/

En la dirección web de esta biblioteca (http://bibliotecas.dipualba.es/pozoca), dentro de los servicios, encontramos el enlace al weblog. Desde este recurso de información se accede al listado de vínculos, donde existe una relación de blogs muy actualizada relacionada con la biblioteconomía, los mensajes más recientes y los archivos de mensajes de meses anteriores, que podemos consultar desde septiembre.

\section{Biblioteca Municipal de La Muela} http://bibliotecalamuela.blogspot.com/

En este weblog podemos encontrar tres apartados fundamentales: el primero son los mensajes más recientes; el segundo, los archivos de mensajes ordenados mensualmente, a los que podemos acceder desde el mes de febrero de 2005, y el tercero, los vínculos o enlaces, casi todos referentes a temas relacionados con $\mathrm{La}$ Muela (Ayuntamiento, Museo del Viento, Museo del Aceite, etcétera).

Fundación Germán Sánchez Ruipérez: Biblioblog y Libraryblog http://www.fundaciongsr.es/biblioblog y http://www.fundaciongsr.es/libraryblog

Hay que mencionar también la última novedad, que acaba de aparecer de manos de esta Fundación: Biblioblog o, en su versión en inglés, Libraryblog. Según la entidad, se trata de un servicio de información sobre bibliotecas que emplea la tecnología de los weblogs para ofrecer noticias de la actualidad biblioteconómica. Nació en mayo de 2003 y desde hace varios meses es mantenido por el Departamento de 


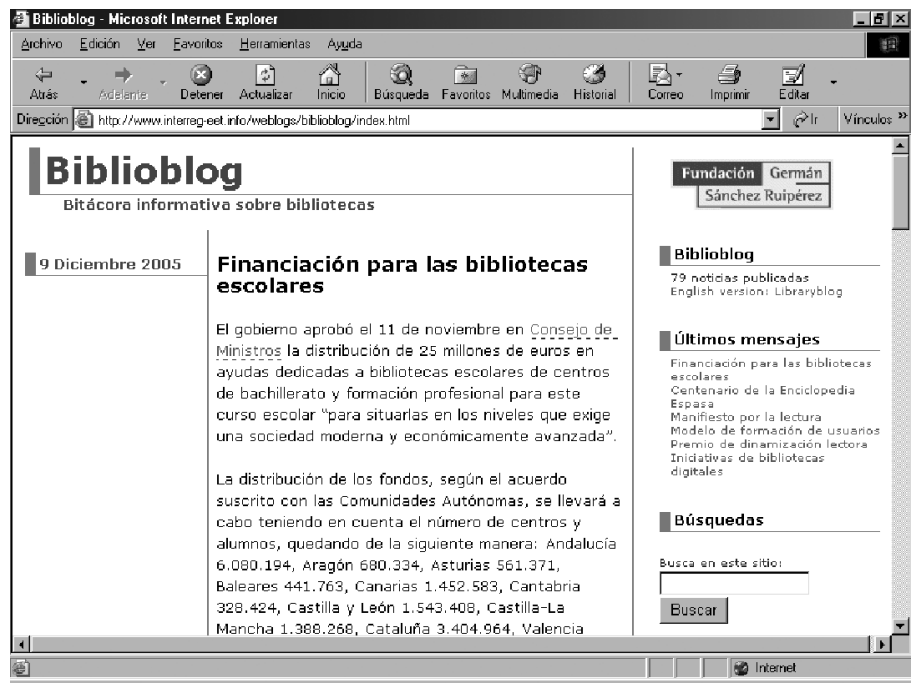

Figura 1. Estructura de Biblioblog.

Análisis y Estudios del Centro de Desarrollo Sociocultural de la Fundación Germán Sánchez Ruipérez, con sede en Peñaranda de Bracamonte (Salamanca).

Libraryblog es la traducción al inglés de la bitácora anterior, y se realiza con la intención de dar a conocer internacionalmente la actividad que se lleva a cabo en España en materia de bibliotecas.

En ambos casos se emplean metadatos DC y sindicación RSS. Gracias a esto, los usuarios pueden estar informados inmediatamente de todas las novedades que se aportan de forma periódica a estas herramientas. Estos dos recursos los podemos encontrar, respectivamente, en http://www.fundaciongsr.es/biblioblog y http://www.fundaciongsr.es/libraryblog.

A continuación exponemos, para tener una visión más amplia, algunos ejemplos de weblogs de bibliotecas fuera de España.

Rowland Institute Library Blog

http://blogs.law.harvard.edu/rihlib/

El bibliotecario de esta institución mantiene este weblog para dar noticia de sitios web, artículos y recursos de interés para investigadores de Rowland, así como material sobre revistas concernientes a bibliotecas científicas. 
http://www.rcpl.info/services/liblog.html

En este blog podemos encontrar, además de las noticias habituales sobre el mundo de Internet y las fuentes de información, un tutorial de búsqueda en el catálogo, acceso al mismo y colecciones digitales. Curiosamente, esta biblioteca de California tiene una versión de la web en español, aunque no del blog.

Roselle Public Library's Blogger Book Club http://bloggerbookclub.blogspot.com

Esta biblioteca tiene un weblog que sirve de soporte a un club de lectura en Internet para niños y adolescentes. Su atractivo diseño es uno de sus principales rasgos.

\section{Monroeville Public Library Teen Summer Reading} http://mplteens.blogspot.com/

Un servicio parecido a la página anteriormente mencionada, pero esta vez dirigido a adolescentes. La biblioteca lo ofrece para los chicos y chicas que quieren expresar lo que le han parecido las lecturas de los libros; además, así les permite relacionarse con otros chicos de su edad y comentar sus aficiones literarias.

The Santa Fe Public Library Blog http://santafelibrary.blogspot.com/

Weblog de la Biblioteca Pública de Santa Fe, en Nuevo México, donde podemos ver cómo se utiliza este recurso para dar a conocer las novedades de la misma, los acontecimientos que se van a desarrollar o un chat sobre los libros.

Hay otros centros que no disponen de weblog propio pero sí ofrecen sindicación de contenidos para algunos servicios en concreto:

Biblioteca de la Australian National University http://anulib.anu.edu.au/epubs/innopacnewbooksrss.html

Da noticia de las últimas adquisiciones de libros y revistas por grandes bloques de materias: ciencias sociales y humanidades, ciencias, derecho, arte y música, etcétera.

Rich Site Services

http://www.public.iastate.edu/ CYBERSTACKS/RSS.htm\#Newbooks

Este sitio web de referencia recoge una gran variedad de instituciones documentales que utilizan sindicación de contenidos para difundir noticias, tutoriales de uso, guías de recursos de Internet, nuevas adquisiciones y servicios de referencia, entre otros servicios.

Scire. $13: 1$ (en.-jun. 2007) 133-144. ISSN 1135-3716. 


\section{Futuro y perspectivas}

En cuanto al futuro de los weblogs, parece que va a estar muy orientado al marketing, ya sea corporativo o personal, y al periodismo participativo.

En un estudio de Jupiter Research se señalaba que estos diarios personales online, surgidos mayoritariamente del esfuerzo personal de los bloggers y sin fines comerciales directos, están captando parte de la creciente publicidad en Internet y son un buen vehículo para el marketing viral, que es el que se propaga a través de recomendaciones directa entre grupos afines de usuarios. Aprovechemos pues esta tendencia y utilicémoslo para publicitarnos de una manera distinta y muy efectiva.

Es un formato en plena expansión y las empresas de Internet lo saben; Google compró el servidor Blogger consciente de la importancia creciente que adquieren cada día. Grandes empresas como HP o Microsoft tienen ya su blog.

Una consecuencia directa de la sindicación de contenidos de una bitácora es que aumenta su "visibilidad" en Internet, es decir, la sitúa en mejores posiciones en los buscadores y le proporciona una difusión añadida al permitir que sea localizada en los buscadores y directorios propios de RSS. Todos conocemos la importancia de estar bien posicionado en buscadores para "ser o no ser" en Internet. El uso de la sindicación de contenidos en unidades de información, independientemente de que esté asociada a un weblog, es, a nuestro parecer, la mayor revolución en relación con los usuarios.

\section{Conclusiones y recomendaciones}

Los gestores de información debemos aprovechar este formato rápido, sencillo y enormemente comunicativo para ofrecer nuestros servicios, ampliando funcionalidades que nos permite la blogosfera.

Queremos concluir destacando las ventajas que esta herramienta nos va a ofrecer en nuestro trabajo diario y que pasamos a comentar.

En primer lugar, puede considerarse un foro de discusión y conseguir el mismo reconocimiento que las listas de distribución y demás foros de intercambio de información, ya que proporciona gran interactividad con los lectores; por ello, utilicémoslo como foro de intercambio de opiniones entre nuestros usuarios y el centro, así como entre ellos mismos.

Además, los weblog se configuran en la actualidad como una herramienta de marketing muy interesante para las unidades de información, ya que debemos intentar fidelizar a los clientes, al igual que el comercio electrónico comenzó a convertir a cada visitante en un cliente habitual. ¡Tomemos ejemplo! Y consigamos esto en las bibliotecas también. Para ello, animemos con el blog a que potenciales lectores se hagan nuevos usuarios de nuestro centro. 
Otra particularidad a resaltar es que también permite una mayor especialización para llegar a cualquier sector de los usuarios, sean, como ya hemos visto en los ejemplos prácticos, adolescentes que utilizan bibliotecas públicas, investigadores de centros de documentación o profesores que usan bibliotecas científicas o universitarias.

Por otra parte, se ha de aprovechar la facilidad de uso y, sobre todo, la de poder poner al día con frecuencia el blog e incorporar fácilmente cualquier noticia. Como ejemplo, uno de los usos más comunes consiste en utilizarlo para dar a conocer las novedades de la biblioteca o centro, ya que, con esta aplicación, los antiguos boletines de noticias que emitíamos en papel o, recientemente, por correo electrónico encuentran un nuevo canal de difusión.

Al ser de actualización frecuente, podemos conseguir que la visita ocasional de los usuarios a nuestra web se convierta ahora, con el weblog, en algo habitual, como por ejemplo los casos que antes comentábamos de crear un área de discusión de libros en línea para pedir que unos lectores recomienden libros a otros.

Asimismo, puede utilizarse como un servicio de apoyo a la comunidad donde nos encontramos situados, con noticias que afecten al entorno local y nos permitan alcanzar una nueva audiencia.

En suma, hemos de concluir afirmando que los bibliotecarios o gestores de información siempre hemos buscado maneras de ofrecer servicios con valor añadido, y creemos que este es uno de ellos. En un entorno caracterizado por la tecnología, los blogs pueden ser instrumentos muy eficaces para captar a todo ese público que navega por Internet.

\section{Referencias}

Alonso, Jaime; Martínez, Lourdes (2003). Medios interactivos: categorización y contenidos. // Díaz Noci, Javier; Salaverría Aliaga, Ramón (coords.). Manual de ciberperiodística. Barcelona: Ariel, 2003. 261-305.

Blood, R. (2000). Weblogs: a history and perspective. // Rebecca's Pocket (7 Sept. 2000). http://www.rebeccablood.net/essays/weblog_history.html (diciembre de 2005).

Blood, R. (2002). The weblog handbook: practical advice on creating and maintaining your blog. Cambridge: Perseus, 2002.

Cambronero, Antonio (2004). RSS, sindicación de contenidos y "planets". // Desde mi bolsillo. http://librodenotas.com/desdemibolsillo/ (diciembre de 2005).

Clyde, L. A. (2004). Weblogs and libraries. Oxford: Chandos, 2004.

Díaz Noci, J.; Salaverría, R. (coords.). Manual de redacción ciberperiodística. Barcelona: Ariel, 2003.

Lasa, R. (2005). Entrevista a José Luis Orihuela. // Noticias de Navarra. (17 de febrero de 2005). http://www.noticiasdenavarra.com/ediciones/2005/02/14/sociedad/navarra/ d14nav11.220841.php (diciembre de 2005).

Scire. $13: 1$ (en.-jun. 2007) 133-144. ISSN 1135-3716. 
Merlo Vega, J. A.; Sorli Rojo, A. (2003). Weblogs: un recurso para los profesionales de la información. // Revista Española de Documentación Científica. 26:2 (abr.-jun. 2003) 227-236. http://exlibris.usal.es/merlo/escritos/weblogs.htm (diciembre de 2005).

Orihuela, J. L. (2004). Los weblogs: de la revolución a la consolidación. // Chasqui: Revista Latinoamericana de Comunicación. 85 (2004). http://www.comunica.org/chasqui/ 85/orihuela85.htm (diciembre de 2005).

Orihuela, J. L. (2005). La revolución de los weblogs. // Perspectivas del Mundo de la Comunicación. 26 (ene.-feb. 2005) 4-5. http://www.unav.es/fcom/perspectivas/pdf/persp26.pdf (diciembre de 2005).

Perrone, J. (2004). What is a weblog? // Guardian Unlimited. http://www.guardian.co.uk/ weblogarticle/0,6799,394059,00.html (diciembre de 2005).

Scott, P. (2005). Blogging: creating instant content for the web. http://library.usask.ca/ $\sim$ scottp/il2001/definitions.html (diciembre de 2005).

\section{Apéndice: enlaces web estudiados}

Base de datos de sumarios de revistas de biblioteconomía y documentación del CSIC:

http://bddoc.csic.es:8080/isoc.do;jsessionid=3F810D154ED2EE8E18DD2337F645E0CD

Bitácoras.com: http://www.bitacoras.com

Bitácoras.net: http://www.bitacoras.net

Blogalia: http://www.blogalia.com

Blogia: http://www.blogia.com

Blogómetro: http://blogometro.blogalia.com

Dialnet: http://dialnet.unirioja.es/

DOIS: http://wotan.liu.edu/dois/

Ecuaderno: http://www.ecuaderno.com

E-Prints: http://eprints.rclis.org/

Sitio web de Laurel A. Clyde sobre weblogs: http://www.hi.is/ anne/weblogs.html 